\title{
Respuestas de política \\ a los problemas de
breza y desigualdad en \\ a los problemas de
pobreza y desigualdad en el mundo en desarrollo
}

\section{Albert Berry \\ Profesor de Economía, Centro de Estudios Internacionales, \\ Universidad de Toronto, Canadá Berry2@chass.utoronto.ca}

El autor sostiene que la efectividad de los instrumentos para aliviar la pobreza depende considerablemente de cómo se defina ésta. El propósito de disminuir la pobreza absoluta implica, principalmente, una discusión del crecimiento, que ha sido la fuente principal de su reducción secular. En cambio, si se define la pobreza en términos relativos, los cambios en la desigualdad pasan a ser la fuente principal de variaciones en su incidencia. En cualquier caso, resulta central la relación entre las dos fuentes potenciales de reducción de la pobreza.

El artículo reseña las implicaciones para el crecimiento y la distribución, primero, de las políticas aplicadas con propósitos de crecimiento y, luego, de aquellas con objetivos distributivos. Finalmente, considera cuáles son los instrumentos más prometedores para reducir la pobreza absoluta o la pobreza relativa y cuán sensitiva puede resultar la combinación óptima de políticas a la manera como se defina la pobreza. 


\section{I}

\section{Introducción}

Según las medidas más usuales del progreso económico - el ingreso per cápita o el poder adquisitivo y la satisfacción de las necesidades básicas - ha habido una notable disminución de la incidencia de la pobreza en el Tercer Mundo, y por lo tanto en el mundo en su conjunto, en los últimos cincuenta años para los que se dispone de datos razonablemente adecuados. Si se toma como base un umbral de pobreza constante de 200 dólares de $1970,{ }^{1}$ la incidencia de la pobreza en el mundo disminuyó de casi $50 \%$ en 1950 a cerca de $25 \%$ en 1977 y probablemente a entre $10 \%$ y $13 \%$ en 1995 , con una baja de más de $40 \%$ en el número absoluto de pobres. ${ }^{2}$ Parte importante de este avance es reflejo del crecimiento acelerado y el bajo nivel de desigualdad de China. Si se excluye China, la incidencia de la pobreza disminuye de manera más pausada, de $36 \%$ en 1977 a cerca de entre $11 \%$ y $15 \%$ en 1995 , mientras que el número de personas en situación de pobreza absoluta baja levemente, quizá entre 5\% y $15 \%$ en todo el período, produciéndose toda o casi toda la reducción después de 1980. Definido en función de un umbral de pobreza más elevado, de mil dólares de 1987, y excluida China, el nivel de pobreza absoluta registró un aumento neto en el período 1950-1995, lo que también se observa en el período más reciente de 1980-1995.

En el mundo no socialista, la baja en la incidencia de la pobreza, definida en función del nivel de ingresos, ha sido intermitente, ha mostrado desigualdad entre regiones y ha estado amenazada por cambios de política y del entorno. En consecuencia, interesa mucho encontrar una receta segura para lograr un tipo de "crecimiento con equidad" que traiga consigo un alivio rápido y generalizado de la pobreza. Pese a que, al menos entre los economistas, hay bastante consenso respecto de algunos de los elementos de una estrategia adecuada para reducirla, hay otros más controvertibles, y dista mucho de haber consenso acerca de

$\square$ Agradezco las observaciones que formularon Gerry Helleiner y José Antonio Ocampo a una versión anterior del presente trabajo. Los errores que subsistan son de mi exclusiva responsabilidad.

1 Y con los valores correspondientes a otros países convertidos a dólares de los Estados Unidos a un tipo de cambio de paridad del poder adquisitivo.

2 Basado en Berry, Bourguignon y Morrisson (1983), y Berry y Serieux (2002). la importancia relativa de los componentes sugeridos. El presente artículo presenta algunas ideas sobre lo que podría surtir efecto y hace hincapié en la necesidad de basar las políticas en un análisis más detenido que hasta ahora del bienestar humano, y de relacionar más estrechamente la definición de la pobreza y la respuesta de política.

Toda discusión sobre el alivio de la pobreza absoluta, cuando ésta es definida por un poder adquisitivo inferior a algún determinado nivel absoluto, es también necesariamente un debate acerca del crecimiento. Podría pensarse que la velocidad con que disminuye la pobreza se determina conjuntamente por el ritmo de incremento del ingreso medio y las variaciones de la distribución del ingreso. Si la distribución se hace más equitativa, contribuye a aliviar la pobreza, y en tal caso el crecimiento habría originado sólo parte del alivio logrado; de lo contrario, "explicaría" la totalidad o más del total de ese alivio. En este sentido mecanicista, la mayor parte, si no la totalidad, de la disminución de la pobreza registrada a lo largo del último medio siglo se debe al crecimiento. En los últimos diez años o más, la agudización de las desigualdades de ingreso ha sido la norma en los países en desarrollo (y también en los desarrollados). En consecuencia, el crecimiento es hoy un camino menos seguro que antes para aliviar la pobreza absoluta, por mucho que se haya convertido en la única fuente de ese alivio.

Conviene distinguir entre la distribución primaria del ingreso, que es aquella que surge del funcionamiento de la economía antes de que haya una redistribución posterior mediante impuestos y transferencias, y la distribución secundaria, que incluye los efectos de éstos. Si se tiene presente tal distinción, podría pensarse que el desafío de mitigar la pobreza involucra i) el crecimiento, ii) los efectos del particular patrón de crecimiento aplicado para la distribución primaria - a veces positivo, a veces negativo- , y iii) la compensación directa de la pobreza, con alguna forma de redistribución para mitigar en parte la pobreza implícita en la distribución primaria del ingreso; esto puede hacerse por el Estado o por conductos privados, como la familia extensa y la comunidad.

Si el ingreso o el consumo relativos son elementos clave de la definición básica de la pobreza - autores como Easterlin (1974), Scitovsky (1976) y Hirsch 
(1976) han sostenido que el consumo es el factor determinante del bienestar en los países industrializados-, es probable que las variaciones de la distribución sean la mayor fuente de cambios en la incidencia de la pobreza, cuando ésta se define como un bajo nivel de lo que los propios afectados conciben como bienestar (más adelante volveremos sobre esto). En todo caso, no hay duda de la importancia de la relación entre ambas fuentes potenciales de reducción de la pobreza, esto es, el crecimiento y el aumento de la equidad. Numerosos teóricos del desarrollo de comienzos de la posguerra hicieron hincapié en que durante un tiempo, el crecimiento bien podría empeorar la distribución del ingreso (Kuznets, 1955). Otros, entre ellos algunos que no creían que en las primeras etapas se deterioraría la distribución y algunos que pensaban que el deterioro no sería lo bastante marcado como para impedir que se beneficiaran los grupos más pobres, adherían a la teoría del chorreo: pese a que el crecimiento no está especialmente destinado a favorecer a los pobres, éstos de todas formas aprovechan parte de sus frutos. En general, la evolución del último medio siglo ha confirmado la teoría del chorreo, por lo menos en el sentido de que se ha logrado una importante disminución de la pobreza, incluso en países a los que no parecía inquietar mayormente este objetivo. La experiencia taiwanesa demuestra hasta qué punto pueden ser complementarios una distribución muy equitativa del ingreso con un crecimiento muy acelerado, incluso en etapas tempranas de desarrollo (Ranis, 1978). Aunque en forma menos espectacular, la trayectoria de otros países es consistente con la teoría. Así pues, el debate sobre el tema crecimiento-distribución ha llevado gradualmente a una aceptación bastante genera- lizada de que lo más probable es que ambos objetivos no entren sistemáticamente en conflicto a menos que la combinación de políticas haya sido desacertada. La gama de experiencias en materia de crecimiento y de distribución indica que las políticas que se apliquen pueden tener mucho peso, es decir, que no toda la variedad de resultados obedece a que las circunstancias exógenas hayan sido diferentes. ${ }^{3}$

Puesto que, especialmente a mayor plazo, el crecimiento es el factor que más contribuye a reducir la pobreza absoluta, medida en función del ingreso, cabe concluir que las políticas que se adoptan — sobre todo por las ventajas de crecimiento que se espera obtener de ellas- también pueden ser los principales factores determinantes de la forma en que varía la pobreza en el tiempo, y que aquellas que se adoptan con fines distributivos cumplan una función menos importante. En la práctica, es posible que algunas de estas últimas empeoren la distribución, ya sea porque gobiernos controlados por los relativamente adinerados rehúyen aplicar políticas que tengan efectos distributivos, ya sea porque aún son objeto de conjetura los efectos redistributivos de muchas políticas.

En la sección II siguiente se examinan las consecuencias que tienen para el crecimiento y la distribución las políticas que se aplican habitualmente con el primero de estos objetivos. En la sección III se hace lo propio respecto de aquellas que se aplican ordinariamente con fines de distribución. Por su parte, la sección IV destaca cuán sensible es la composición del conjunto óptimo de políticas a la forma en que se define la pobreza, mientras que la sección $\mathrm{V}$ contiene algunas conclusiones generales acerca de las "mejores políticas".

\section{II}

\section{Efectos en el crecimiento y la distribución derivados de las políticas orientadas principalmente al crecimiento}

La importancia de la formación de capital físico, la acumulación de capital humano y el cambio tecnológico como fuentes de crecimiento no ha sido objeto de un estudio serio. Lo único que se discute gira en torno a su importancia relativa y cómo ella varía según las circunstancias y a la mejor manera de avan- zar en cada uno de estos procesos. A este último respecto, naturalmente son pertinentes la política fiscal

\footnotetext{
${ }^{3}$ La historia de las ideas sobre la pobreza y su relación con el crecimiento, así como muchas cuestiones relacionadas con las políticas para enfrentar la pobreza se examinan en Lipton y Ravallion (1995).
} 
y la política comercial. Las repercusiones distributivas de los tres procesos son más complejas y posiblemente dependan mucho de los pormenores de cada caso.

\section{Política macroeconómica conservadora}

Hay consenso en que una inflación desenfrenada es perjudicial para el desarrollo; lo que no está tan claro es si una inflación baja (por ejemplo, de un solo dígito) conduce a más crecimiento que una inflación baja de dos dígitos, y en qué circunstancias. Al parecer, la marcada preferencia actual por tasas bastante bajas de inflación se basa principalmente en ideas conservadoras, cuyas predicciones pueden por cierto resultar acertadas, y en la impresión de que en un mundo que evoluciona hacia una mayor integración, una inflación elevada plantea mayores problemas. Sin embargo, al parecer la hiperinflación sí tiene efectos negativos en la distribución, por mucho que puedan ser de corta duración, y en todo caso no es factor que determine la desigualdad. Además, a menudo se sostiene que el "impuesto inflación", que se produce incluso con grados de inflación intermedios, es de carácter bastante regresivo, en cuyo caso lo más probable es que una política monetaria expansiva también lo sea. Sin embargo, la evidencia es débil y teóricamente no hay grandes razones para prever que los efectos se inclinen en una u otra dirección, salvo que el carácter expansivo de la política monetaria y fiscal y la distribución de los beneficios netos que producen (impuestos, gastos del gobierno, acceso al crédito) estén directamente relacionadas. Existe la impresión bastante generalizada de que las bajas tasas de interés real que suelen acompañar a tasas de inflación altas tienden a producir una distribución del crédito más concentrada que las que se acercan más a los niveles de mercado (Fry, 1988, p. 163), pero en este caso la evidencia continúa siendo escasa. Las hipótesis sobre los efectos distributivos de una política fiscal expansiva en contraposición a una restrictiva son más variadas, y dista de haber consenso al respecto (Whitehead, 1996). En general, pese a que no hay razones fundadas para pensar que estas políticas tienen efectos directos importantes en la equidad, las modalidades de crédito, impuestos y gasto público que las acompañan pueden tener bastante gravitación, si bien en formas que aún no se conocen bien.

Las teorías recientes sobre la histéresis de las condiciones sociales en el curso del ciclo económico ponen aún más de relieve la complejidad de los efec- tos de la política macroeoconómica en la distribución y la pobreza. De acuerdo con ellas, se sostiene, por ejemplo, que la pobreza, el capital humano y el empleo no se ven afectados simétricamente por el ciclo descendente y la posterior reactivación que en conjunto constituyen un ciclo económico, puesto que los beneficios de la reactivación generalmente son menores que las pérdidas del ciclo descendente. En consecuencia, los ciclos económicos no son sucesos neutros: cuando el PIB regrese a su nivel anterior a la recesión, por lo general no sucederá lo mismo con otras variables; el comportamiento cíclico puede tener costos permanentes en el sentido de mantener esas variables por debajo de los niveles que habrían alcanzado si el PIB hubiera evolucionado de manera menos inestable. Estos planteamientos se relacionan con la idea de que en los casos en que la estabilidad de precios, en sí deseable, se logra a expensas de la estabilidad del crecimiento del producto, los efectos netos de redistribución pueden ser negativos (Ocampo, 2002).

Según lo revelan los estudios antes citados, la importante contribución directa a la satisfacción de la gente que significa el hecho de tener empleo hace que todo conjunto de políticas que conduzca a un desempleo elevado - por ejemplo, una política monetariofiscal restrictiva que tenga por objeto lograr el equilibrio de la balanza de pagos- es doblemente problemático. Las políticas de esta índole probablemente sólo se justifiquen en caso de que se requiera un desempleo presente elevado para asegurar que en el futuro sea bajo.

\section{Las políticas de ahorro e inversión}

Para lograr un crecimiento realmente acelerado, el ahorro tiene que ser alto y su utilización eficaz. Tanto la correlación comparativa entre países de las tasas de ahorro e inversión con la tasa de crecimiento, como el hecho de que todos los países de rápido crecimiento de Asia oriental experimentaron incrementos espectaculares de sus tasas de ahorro como parte de la aceleración del crecimiento (Banco Mundial, 1993), ponen de relieve la importancia decisiva de esta variable. Por desgracia, sigue habiendo una gran incertidumbre en cuanto a qué factores contribuyen a aumentar el ahorro en qué tipos de países. En Asia oriental, parece haber ayudado la baja carga demográfica (Bloom y Williamson, 1998); al parecer, cuando las tasas de interés real son positivas y el sistema financiero es firme aumenta el ahorro financiero (Mason, 1988), pero no resulta claro si estos factores influyen o no en el 
ahorro total (Masson, Bayoumi y Samiai, 1998, p. 497; Akyüz, 1995). El crecimiento acelerado parece contribuir a que la tasa de ahorro sea elevada, de modo que posiblemente el principal problema sea alcanzar esa primera racha de crecimiento acelerado.

$\mathrm{Si}$ en la práctica el potencial de ahorro se limitara a los ricos - digamos, la clase capitalista - habría una clara relación inversa entre el crecimiento acelerado y la distribución equitativa del ingreso. No siempre sucede así, según queda ilustrado por experiencias como la taiwanesa, pero bien puede ser que esta relación inversa esté ausente sólo en condiciones algo limitadas. Sin duda es mejor lograr que un pequeño segmento de la población ahorre mucho que no lograr ahorro alguno, en especial cuando se trata de aliviar la pobreza definida en términos absolutos. Cuando son los empresarios, algunos de los cuales no son particularmente ricos, los que concentran el ahorro, la situación resulta más aceptable, puesto que la distribución del gasto de consumo es bastante menos desigual que la del ingreso.

\section{La acumulación de capital humano}

No obstante que, en general, se reconoce la importancia de la acumulación de capital humano para el crecimiento (Schultz, 1961) y que los mecanismos que vinculan a ambos se han estudiado desde diversos puntos de vista, hoy se sabe muy poco más que hace algunos decenios acerca del papel que desempeña el capital humano en el proceso de crecimiento. Se ha demostrado que las regresiones de Mincer, que desde hace mucho son el testimonio más ampliamente utilizado de los beneficios sociales que traen consigo la educación y la capacitación, son muy vulnerables a los problemas de especificación, en particular al sesgo de las variables omitidas y al sesgo de la agregación (Rosenzweig, 1999; Schultz, 1989); el credencialismo, cuya posible importancia cuantitativa ha resultado más difícil de demostrar, continúa arrojando dudas sobre la interpretación más sencilla de Mincer sobre las diferencias de ingresos y sugiriendo que ellas adolecen de un sesgo ascendente (Dore, 1976). Por otra parte, las externalidades positivas relacionadas con el proceso de innovación imitable, con la adquisición de conocimientos a partir de las personas que tienen mayor educación, etc., también podrían ser importantes y tenderían a producir un sesgo descendente en las estimaciones estándares. En el mejor de los casos, las estimaciones basadas en la regresión de Mincer dan una idea muy burda de los beneficios sociales de la educación; es probable que haya que partir de la base de que una tasa de rentabilidad estimada de $15 \%$ significa que la tasa real fluctúa entre 5\% y $20 \% .{ }^{4}$ Para reducir el rango de incertidumbre ${ }^{5}$ sería necesario que convergieran los resultados de las regresiones de Mincer y la evidencia que ofrecen otras metodologías. Siendo que actualmente están en discusión diversos tipos de capacitación práctica (incluida la actualización profesional periódica), que tienen que ver en diversas formas con el alivio de la pobreza, resulta desalentador que el análisis de la función del ingreso tenga dificultades aún más evidentes para dilucidar los beneficios de estas formas de capital humano que para esclarecer los de la educación académica.

Las estimaciones de Mincer han sido motivo de optimismo no sólo porque generalmente han asignado un valor mediano a alto a la rentabilidad social global de una mayor acumulación de capital humano, sino también porque indican que en el caso de la enseñanza básica esa rentabilidad es particularmente elevada. Esto respalda la convicción de que aumentar el capital humano de quienes se encuentran próximos al extremo inferior de los perfiles de educación y de ingresos es un instrumento útil para luchar contra la pobreza, quizá el más importante de todos. Pero se sigue sabiendo poco sobre qué medidas reducirían efectivamente la brecha educacional — para determinarlo habría que saber, por ejemplo, cómo reaccionarían los que se encuentran en el extremo superior de esa brecha- y la forma en que puedan evolucionar en el futuro las diferencias de ingresos relacionadas con la educación. La evidencia empírica que vincula el progreso en materia de capital humano con mejoras en el crecimiento y la distribución es demasiado débil como para que pueda sustituir un conocimiento más profundo de lo que sucede dentro de esta "caja negra". A la luz de la aparente importancia de las destrezas vinculadas al conocimiento de la informática y la revolución de la información, y de los incrementos de las diferencias de ingreso según nivel educativo observados en los últimos diez años o más (Robbins, 1995), es muy posible que en el mediano plazo hayan aumentado en términos relativos los beneficios privados de un nivel

\footnotetext{
${ }^{4}$ Estimaciones como las de Behrman y Deolalikar (1993) sugieren la posibilidad de que los verdaderos efectos de la educación en la productividad no superan un $40 \%$ de lo que señalan las regresiones simples de Mincer.

${ }^{5}$ Pritchett (1996) describió crudamente la confusión reinante en esta materia. Se han realizado demasiados estudios simplistas y no ha habido suficiente preocupación por los numerosos problemas metodológicos.
} 
educativo más alto, en contraposición a los derivados de niveles educativos menores. A medida que en el contexto de la globalización se facilita la migración ilustrada, resulta cada vez más difícil conservar en el país a las personas capacitadas; puede ser que la opción de emigrar sea uno de los factores responsables de la creciente diferencia de ingresos que se observa en los países en desarrollo (Hamada y Bhagwati, 1975). Estas consideraciones podrían significar que la estrategia óptima para promover el crecimiento en materia de capital humano probablemente ya no mejore la distribución ni alivie la pobreza, por mucho que haya podido hacerlo en algún momento del pasado.

Las razones para fomentar una enseñanza básica generalizada y de buena calidad siguen siendo poderosas, ya que las ventajas que acarrean la alfabetización y los conocimientos elementales de aritmética son evidentes y este nivel de instrucción es indispensable para permitir que las personas avancen a los niveles superiores. Sin embargo, la actual ambigüedad de la información sobre los beneficios que confiere la educación en general tornan aún más urgente prestar mayor atención a la calidad de la enseñanza básica. Además, y como sucede con la mayoría de los demás mecanismos en estudio, es probable que en la relación entre capital humano y pobreza influya la forma en que se defina la pobreza. De más está decir que éste es el caso si se utiliza una definición vinculada a las "necesidades básicas", en la cual la educación ocupa un lugar importante.

\section{El cambio tecnológico}

La importancia del ahorro y la inversión como fuentes de crecimiento no se pone en duda; en cambio, sí se discute cuáles son las tecnologías nuevas que contribuyen al crecimiento; la teoría sostiene que las tecnologías demasiado modernas o con uso intensivo de capital tal vez no lo hagan, y que las tecnologías intermedias o "apropiadas" son una apuesta más segura. Sin embargo, la evidencia empírica a este respecto es limitada y en su mayor parte indirecta, y hay grandes dudas de que se disponga de suficientes tecnologías apropiadas.

Los efectos distributivos del progreso tecnológico también son poco claros. La teoría apunta fuertemente a que la tecnología moderna - porque crea pocos empleos por unidad de inversión-, tenderá a empeorar la distribución de los ingresos entre el capital y la mano de obra y que, en los casos en que esa tecnología requiera a la vez mano de obra muy calificada, empeorará también la distribución del ingreso entre trabajadores menos calificados y más calificados. En parte porque carecemos de indicadores del ritmo o la modalidad del cambio tecnológico (sí los tenemos respecto de la formación de capital humano, el ahorro, etc.), ha sido difícil formarse una impresión real de la validez de estos supuestos teóricos. El cambio tecnológico puede ser un peligro para la distribución equitativa $y$, en consecuencia, para el alivio de la pobreza, ya sea por su sesgo hacia el uso del capital o de la mano de obra calificada o porque las empresas que están en condiciones de adoptarlo son solamente las que ya tienen mejor situación (por ejemplo, porque son más grandes). Algunos cambios en la agricultura pueden tener efectos adversos en la distribución debido a ambas razones, por ejemplo, cuando el uso de tractores no sólo disminuye los costos en los grandes predios en formas que los predios pequeños no pueden imitar, sino que además desplaza la mano de obra y empuja a más trabajadores hacia las granjas pequeñas. ${ }^{6}$ También en otros sectores, el tamaño de las empresas que adoptan nuevas tecnologías, así como las tecnologías ya existentes en ellas, influyen en el impacto final de esa adopción (James, 1998; Berry, 1998a).

Algunos aspectos del proceso de cambio tecnológico son más exógenos a los distintos países en desarrollo, e incluso al conjunto de ellos, que las demás fuentes de crecimiento antes examinadas. En general, se observa que alrededor de $97 \%$ de la inversión en investigación y desarrollo se produce en los países industrializados del norte (Banco Mundial, 1999). En consecuencia, es posible que a los países en desarro1lo, en especial si están ligados estrechamente al sistema económico mundial, les resulte muy difícil protegerse de algunos de los efectos adversos de la modalidad actual de cambio tecnológico a nivel mundial. Aún así, mucha de la evolución de las prácticas tecnológicas se ve afectada por la situación y las políticas internas. No obstante que los grandes avances en la investigación agrícola generalmente provienen del exterior, para adoptarlos eficazmente, y en especial para que los adopten los agricultores más pequeños, es indispensable complementar las investigaciones internacionales con el esfuerzo local (Evenson, 1975). En otros sectores existen políticas que contribuyen clara-

\footnotetext{
${ }^{6}$ El Banco Mundial reconoció más bien tardíamente este peligro a comienzos del decenio de 1970. Contribuyó a ello un amplio estudio sobre la utilización de tractores en Pakistán.
} 
mente a que las empresas más pequeñas introduzcan mejoras tecnológicas (Levy, Berry y Nugent, 1999), las que por lo general favorecen más el empleo que las adoptadas por empresas más grandes.

\section{La orientación hacia afuera y otras políticas en pro del mercado}

Los partidarios de un comercio más libre, de menos intervención del gobierno y, en general, de políticas económicas más favorables al mercado han sostenido que un cambio en ese sentido elevará la tasa de crecimiento, no sólo mediante los efectos de eficiencia en la asignación de recursos (Feder, 1983), sino también porque el mayor contacto con fuentes externas contribuirá a elevar al ahorro interno, el ahorro externo (entradas de capital) y el ritmo del cambio tecnológico (Rodrik, 1995, pp. 2933-2934). No cabe duda de que cada uno de los factores mencionados se presenta con cierta frecuencia. Por otro lado, hay que mencionar los efectos adversos de la inestabilidad de la entrada y salida de capitales que ha traído consigo la liberalización de los movimientos de capital, y el efecto negativo que puede producirse en la inversión cuando los mercados más libres disminuyen la certeza de los beneficios de algunas inversiones. Además, en la medida en que la presencia de los efectos — de aprender por la práctica- que se plantea en el argumento de las industrias incipientes justifique algún grado de protección, la imposibilidad de brindar protección puede impedir que los países ayuden a industrias nacientes que podrían llegar a ser competitivas. Queda por verse en qué circunstancias han de predominar los efectos positivos de la orientación hacia fuera. Dado que, con escasas excepciones, la adopción de políticas favorables al mercado todavía no ha tenido efectos positivos inmediatos o amplios en el crecimiento de los países en desarrollo, el caso no está cerrado. Puede ser que esos efectos sean en definitiva tan positivos como esperan los partidarios de la teoría, pero que se requiere un período de gestación más bien largo para que los beneficios se manifiesten en plenitud, o bien puede ser que simplemente no había razones para ser optimistas. El nivel de análisis que sirvió de base para abogar por el cambio de política distó mucho de ser ejemplar. ${ }^{7}$

\footnotetext{
${ }^{7}$ Por ejemplo, no obstante que se utilizaron ampliamente regresiones comparadas entre países que indicaban que la orientación hacia afuera y el crecimiento estaban directamente relacionados, no se prestó mucha atención al hecho de que la presencia de un efecto positivo en los distintos países no significa necesaria o generalmen-
}

Tampoco la teoría económica ayuda mucho a esclarecer cuáles son las políticas comerciales que darían mejores resultados en los países en desarrollo, ya que depende demasiado de las hipótesis que se incorporen en la teoría. Cuando los inversionistas tienen marcada aversión al riesgo y el aprendizaje empírico es importante, tal vez haya que proteger a las industrias incipientes a fin de estimular inversiones que contribuyan al crecimiento. Si los países en desarrollo tuviesen éxito en concebir y efectuar con eficacia una intervención de la política, el libre comercio seguramente no sería su primera opción. El hecho de que estos países a menudo no logren proteger a las industrias incipientes en forma cercana a la que contempla la teoría, hace que el comercio sustancialmente libre resulte más atractivo como segunda opción.

Es muy posible que las políticas orientadas hacia afuera sean las más adecuadas para los países pequeños, pero que lo sean menos para los grandes. Cuando a partir del decenio de 1950 Perú adoptó estrategias de industrialización basadas en la sustitución de las importaciones, obtuvo resultados que parecen haber sido mucho menos satisfactorios que los de Brasil, que entre 1945 y 1980, aplicando vigorosas políticas de industrialización sustitutiva, creció más que casi cualquier otro país del mundo (Maddison, 1992). El crecimiento que se logra promoviendo la inversión de las empresas transnacionales en exportaciones que hacen uso intensivo de mano de obra es mucho más importante para los países pequeños que para los seis o más países en que vive la mayor parte de la población del mundo en desarrollo.

Las consecuencias distributivas de la liberalización de los mercados, incluidos el comercio y la circulación de capital, son objeto de gran controversia. La mayoría de los teóricos prevén efectos de HeckscherOhlin positivos basándose en que, primero, los países en desarrollo generalmente tienen ventajas comparativas en materia de bienes y servicios con uso intensivo de mano de obra, por lo cual la mayor libertad de comercio debería aumentar la demanda relativa de mano

te que tenga un efecto positivo para el Tercer Mundo en su conjunto - ejemplo clásico de la falacia de la composición. Este tema es examinado en el caso de las exportaciones de productos básicos, entre otros, por Maizels (1994) y en el de las manufacturas por Faini, Clavijo y Senhadji-Semlali (1992) y por la UNCTAD (1996). Un defecto aún mayor, porque puede pasar inadvertido al menos experto, ha sido la falta de cuidado en la medición de la "orientación hacia afuera". Las medidas alternativas, cada una de las cuales parece tener su propia lógica, no están bien correlacionadas entre ellas. 
de obra y por lo tanto mejorar la distribución del ingreso, y segundo, en que la mayor libertad de comercio a menudo acarrea una mayor demanda de productos agrícolas cuyas exportaciones son desalentadas indirectamente por las políticas de industrialización basadas en la sustitución de las importaciones, que apuntan principalmente a proteger a la industria manufacturera. Entre los diversos argumentos en contrario, cabe señalar que en muchos países en desarrollo y también en países desarrollados, la reciente oleada de globalización y de liberalización del comercio y de circulación de capitales ha coincidido con una creciente desigualdad (Berry y Stewart, 1997), por lo que cabe dudar que los efectos de Heckscher-Ohlin sean tan marcados como lo previsto. ${ }^{8}$ En algunas regiones, por ejemplo en América Latina, puede ser que ello se explique porque a nivel mundial las ventajas comparativas de estos países no radican en bienes y servicios que hacen uso intensivo de mano de obra no calificada, sino en bienes que hacen uso intensivo de recursos naturales, o de mano de obra relativamente calificada, o de ambos. En general, el hecho de que las empresas que se dedican en gran medida al comercio internacional sean más grandes ${ }^{9}$ que las que no lo hacen y que, ade- más, tiendan a utilizar tecnologías modernas y de mayor densidad de capital, sugiere que a medida que aumente la proporción del producto que se transa internacionalmente, la distribución tenderá a deteriorarse.

Sumando los aún dudosos efectos de la mayor libertad del comercio, sobre el crecimiento económico, a las tendencias negativas en materia de distribución que a menudo han coincidido con los cambios de política, resulta evidente que por ahora no puede sostenerse que la mayor libertad de los mercados traiga consigo un importante alivio de la pobreza. Quizá el resultado más probable sea un pequeño efecto positivo en el crecimiento, junto con uno negativo a mediano plazo en la distribución, con lo cual a la larga se observará un pequeño efecto, negativo o positivo, en la tasa de disminución de la pobreza absoluta. Sin embargo, dados nuestro escaso conocimiento de los mecanismos en juego y la trayectoria empírica más bien confusa recorrida hasta ahora, la gama de efectos posibles es muy amplia. En una sociedad que atribuye apreciable importancia al ingreso relativo, si el efecto en la distribución es muy negativo, el efecto de este cambio de política en la pobreza podría ser marcadamente negativo.

\section{III}

\section{Efectos en la distribución y el crecimiento de las políticas fundamentalmente distributivas}

Tal como sucede con los componentes principales de las políticas que apuntan de preferencia al crecimiento, cada una de las políticas orientadas a la distribución que se examinan enseguida, además de tener los efectos distributivos por los cuales suelen aplicarse, pueden influir significativamente en el crecimiento.

\footnotetext{
${ }^{8}$ Diversos estudios empíricos han llegado a conclusiones más positivas. Así, Londoño y Székely (1998), basándose en regresiones comparativas entre países de América Latina, concluyeron que la reforma del comercio había tenido efectos positivos en la distribución, mientras que la reforma laboral había repercutido negativamente en la distribución a nivel de hogares. Morley (2000) también encontró que las consecuencias de las reformas del comercio eran negativas. Pese a que estimaron que las reformas tendrían efectos positivos, Londoño y Székely llegaron a la conclusión de que por sí solas no mejorarían mucho la desigualdad ni la pobreza, salvo que tuvieran por consecuencia reducir la desigualdad en materia de educación y ampliar el acceso a otros activos productivos.

${ }^{9}$ Más allá del sector en que operen.
}

\section{Las políticas de redistribución de los activos}

La manera más evidente de mejorar la distribución del ingreso es mediante la redistribución de los activos productivos. Cuando se trata del capital humano, esta redistribución sólo puede hacerse de manera gradual a lo largo del tiempo, puesto que no es factible expropiar y redistribuir de inmediato. En el caso de los activos físicos, la redistribución es posible, pero realmente viable tan sólo en el caso de la tierra, esto es, mediante la reforma agraria. No hay duda de que si las condiciones son favorables, ella puede tener efectos importantes y perdurables en la distribución del ingreso. Al respecto, el ejemplo más conocido es el de la provincia china de Taiwán, donde la reforma, realizada cuando esta economía seguía siendo primordialmente agrícola, al parecer produjo una marcada baja de la desigualdad (Kuo, 1983, pp. 94 a 97). Posteriormente, 
su trayectoria ha sido excepcionalmente positiva, a la vez en materia de ingreso y de distribución. Al parecer, la igualdad inicial contribuyó a perpetuarse puesto que ayudó a una distribución muy equitativa de las posibilidades de educación, a un crecimiento dinámico de la pequeña empresa, primero en las zonas rurales y luego en las urbanas, y a una mayor participación política de los representantes de la pequeña empresa. Sin embargo, como son pocos los países en que se ha realizado una reforma agraria significativa, las experiencias son demasiado escasas como para poder pronunciarse sobre las circunstancias en que los efectos serían todo lo positivos que parecen haber sido en Taiwán o en la República de Corea. También es evidente que sólo una situación política muy excepcional abre el camino a una reforma agraria profunda. En consecuencia, esta reforma no parece estar destinada a ser un componente importante de las estrategias de alivio de la pobreza, pese a que en principio podría ser el instrumento más promisorio para muchos países menos desarrollados.

Últimamente se ha prestado alguna atención a las posibilidades que ofrece la reforma agraria basada en el mercado, por la cual la tierra se transfiere reemplazando la expropiación por subsidios para ayudar a los pequeños agricultores a adquirirla. Desde el punto de vista económico, esta reforma tiene perspectivas halagüeñas (Deininger, 1999), aunque si se aplica sin recurrir a la reforma agraria confiscatoria tradicional lo más probable es que en la práctica no pase de tener un efecto secundario en la estructura del agro. Complementada por una reforma tradicional de esta naturale$\mathrm{za}$, puede tener más valor, en parte porque el "efecto amenaza" puede volver más dúctiles a los terratenientes que enfrentan la posibilidad de expropiación. Para ello al menos hay que eliminar las limitaciones legales a la subdivisión de la tierra (Lipton, 1993, p. 651). Un valioso mecanismo de apoyo para que la legislación agraria o crediticia ayude a los pobres es eliminar los subsidios que favorecen a los grandes terratenientes o sus cultivos, medida que puede verse estimulada por las restricciones fiscales que enfrentan tantos países. En el noreste de Brasil la baja de los subsidios contribuyó a que los ricos no se vieran estimulados a subvertir la reforma, aunque en ese caso también se adoptaron otras medidas positivas.

En muchos países, la gente que vive en zonas de bosques ha confrontado la posibilidad de ser expulsada de sus bases de recursos naturales bajo presiones de otros actores, a veces empresas madereras privadas y a veces el Estado. En este sector, al igual que en la agricultura, la buena distribución y la producción eficiente a menudo van de la mano, como lo demuestra el éxito logrado en varios estados de la India (Kant, 1996) y en otros lugares (Brightman, 1987) con los bosques administrados por la comunidad, en los que se produce una amplia gama de bienes madereros y no madereros. En 1988 el Gobierno de la India, reconociendo su fracaso en la producción y la distribución, finalmente abandonó su antigua política de excluir a esas personas de la gestión de los bosques (Kant y Berry, 1999).

\section{La política de apoyo a granjas pequeñas, micro, pequeñas y medianas empresas}

Desde el punto de vista político, las formas de apoyo a la pequeña empresa en la agricultura y otros sectores, que en la mayoría de los países en desarrollo da empleo a la mayor parte de la fuerza de trabajo, son mucho más viables que las políticas de redistribución de los activos. En la medida en que incremente la producción y la productividad de tales empresas, ese apoyo eleva directamente el ingreso de muchos de los más pobres. Otra de sus ventajas consiste en que, cualquiera sea la industria, las empresas más pequeñas generalmente hacen uso más intensivo de mano de obra que las empresas mayores, de modo que su presencia eleva la demanda total de mano de obra respecto de la que habría existido si hubieran sido reemplazadas por empresas más grandes. Pese a que las empresas pequeñas a menudo generan bajos ingresos, su nivel de productividad (esto es, producto por unidad de insumos escasos) suele ser alto, aunque variado. Las empresas medianas con frecuencia logran una mayor productividad total de los factores que las otras categorías y, en consecuencia, contribuyen al crecimiento y al logro de ingresos elevados (Ho, 1980). Las empresas pequeñas y medianas contribuyen a una distribución más equitativa puesto que en ellas las proporciones de los factores generalmente se aproximan a aquéllas de la economía en su conjunto.

Elevar la productividad de las granjas pequeñas y de las microempresas tiene efectos inmediatos en la pobreza. En el primer caso, el éxito obedece a causas claras, como el aumento de rendimiento que ocurre en la revolución verde. En el caso de las microempresas no agrícolas, gran parte de los esfuerzos han apuntado a mejorar el acceso al crédito, partiendo de la base de que ello se traducirá en una mayor productividad y, por lo tanto, en mayores ingresos. En este caso, lo importante es asegurar la sustentabilidad de las instituciones de crédito, cosa que en la mayoría de las experiencias 
no se ha logrado (Santor, 1999). En cuanto a otro punto igualmente importante, si la relación beneficio-costo social es adecuada, la evidencia es más difícil de encontrar y hasta ahora continúa siendo dudosa. ${ }^{10} \mathrm{Si}$ los programas de apoyo disponibles no tienen grandes perspectivas de aumentar la productividad colectiva de la microempresa, se planteará el dilema de si ese apoyo es más o es menos eficaz para disminuir la pobreza que las medidas focalizadas que involucran alguna forma de transferencia a los pobres.

Si no es factible lograr que las empresas muy pequeñas aumenten considerablemente la productividad, otra manera directa de aliviar la pobreza es estimular una expansión acelerada de las empresas medianas y pequeñas, lo que con el tiempo repercutirá en el mercado de trabajo y creará empleos para los que actualmente se desempeñan en el sector de microempresas, que es menos productivo (Berry, 1998b). Según el país de que se trate, este efecto en la pobreza puede producirse muy rápidamente o con algún rezago.

\section{Políticas para el alivio directo de la pobreza}

Todas las sociedades tienen maneras de aliviar la pobreza de quienes carecen de medios adecuados a partir de sus propios esfuerzos u oportunidades. En los países en desarrollo, la mayoría de esos mecanismos involucran a la familia, incluida la familia extensa, o a la comunidad. Estos países han estado elaborando técnicas de focalización desde hace más o menos una década; los resultados estadísticos, sin ser decisivos, ofrecen buenas perspectivas (Grosh, 1995) de que con más experimentación y perfeccionamiento esas técnicas podrán contribuir cada vez más, al menos en algunos países, al alivio de la pobreza en los próximos decenios. ${ }^{11}$

Entre los países en desarrollo, algunos de los mayores ingresos (entre ellos unos latinoamericanos) avanzan hacia instituciones tan corrientes en los países desarrollados como el seguro de desempleo. Estas también deberán pasar inevitablemente por un período de experimentación antes de que se determine claramente la función que pueden y deben cumplir. Lo mismo puede decirse de los diversos programas públicos de empleo que han servido de "redes de seguridad" en varios países en los últimos decenios, desde el estado indio de Maharashtra a Chile bajo Pinochet. Estos programas pagan un salario lo bastante bajo como para asegurar que lo acepten únicamente los necesitados y tratan de generar beneficios sociales en la forma de infraestructura (mantenimiento de caminos, construcción de escuelas, etc.).

\section{IV}

\section{Las definiciones de la pobreza y las prioridades de política}

Los errores de interpretación cometidos en el pasado sobre la forma en que se produce el desarrollo son al menos equiparables con el simplismo de algunas definiciones de la pobreza. Desde hace mucho tiempo, científicos sociales distintos de los economistas, en especial sociólogos y sicólogos, han hecho hincapié en

\footnotetext{
${ }^{10}$ Aunque en muchos casos resulta claro que a los beneficiarios de créditos les ha ido mejor que a quienes no los han obtenido, queda por resolver si las ganancias de los primeros han sido a expensas de las pérdidas de los segundos, escenario que no deja de ser plausible en casos como el del comercio minorista en pequeña escala, cuya estructura es de competencia monopólica y en que el tamaño tota del mercado puede ser bastante reducido. Morduch (1998) toca aspectos interesantes en un estudio sobre las principales instituciones de microcrédito de Bangladesh.
}

que el sentimiento de privación que experimentan las personas se relaciona estrechamente con un ingreso y un poder adquisitivo más bajos que los de otros y con el sentimiento de inferioridad que esto origina, particularmente en sociedades competitivas que juzgan a las personas por su éxito relativo. La atención preferente prestada por los economistas a medidas del bienestar basadas en el ingreso absoluto posiblemente no haya

\footnotetext{
11 Ocampo (2001, p. 25) observa que en Brasil y Chile, dos de los tres países de América Latina que en el decenio de 1990 exhibieron la disminución más marcada de la pobreza rural (el tercero fue Panamá), la reducción estuvo estrechamente vinculada con la focalización de las transferencias y los subsidios estatales en los sectores más pobres.
} 
obedecido más que a un mero prejuicio, no respaldado en definitiva por la evidencia más directa. En otras palabras, cuando se pregunta directamente a las personas que viven en los países industrializados (donde se suele efectuar esta clase de estudios) por su felicidad y sus correlatos, el ingreso absoluto parece desempeñar un papel muy inferior a lo que indicaría la teoría económica: es moderadamente significativo cuando se compara a personas pertenecientes a diversos niveles de la escala de ingresos en un momento determinado, pero lo es menos - algunos autores lo consideran insignificante - como factor de la variación media del bienestar societal a lo largo del tiempo, incluso cuando el promedio de ingresos ha aumentado de manera apreciable (Easterlin, 1974; Scitovsky, 1976; Oswald, 1997). La mayoría de estos estudios revela que la variable económica que ejerce mayor influencia es el empleo, ya que las personas que tienen trabajo son mucho más felices que las que carecen de él. ${ }^{12}$ Las personas instruidas son más felices que las que carecen de educación, los que trabajan por cuenta propia más que los empleados, y los jubilados más que los económicamente activos. El significado final de toda esta evidencia es todavía una incógnita; en especial, es apremiante examinar más a fondo el papel secundario que parece desempeñar el ingreso. ${ }^{13}$

Podría pensarse que en los países en desarrollo de menores ingresos éstos fueran un factor más importante en la determinación del grado de felicidad, y los datos disponibles así lo indican. Frank (1997, p. 1834) observa que los estudios más minuciosos encuentran en las series cronológicas una clara relación entre el bienestar subjetivo y el ingreso absoluto, a niveles bajos de ingreso absoluto. Allí donde la mayoría de las personas carece de un mínimo adecuado en materia de vivienda y alimentación, el ingreso adicional eleva el bienestar subjetivo en forma significativa y perdura-

\footnotetext{
${ }^{12}$ Cabe suponer que esto se debe en parte a que a las personas que no son felices les cuesta encontrar trabajo, pero estudios longitudinales realizados por sicólogos han demostrado que ésta no es la única causa (Oswald, 1997, p. 1822).

${ }^{13}$ No obstante que el aumento del ingreso medio parece no conducir a aumentos equiparables de felicidad, efectivamente hay una importante relación transversal entre felicidad e ingreso. Datos de Europa correspondientes al período 1975-1986 señalan que $18.8 \%$ del quintil inferior declara ser "muy feliz", en comparación con $28.4 \%$ del quintil superior, mientras que un $26.7 \%$ del quintil inferior dice "no ser demasiado feliz", en comparación con un $13.1 \%$ del quintil superior (Di Tella, MacCulloch y Oswald, 1996). Parte de esta correlación se debería a que los grupos de bajos ingresos registran tasas más elevadas de desempleo. A lo largo del tiempo, la felicidad de los desempleados oscila mucho más que la de los empleados, por razones aún no explicadas.
}

ble (Diener y Diener, 1995). En los países extremadamente pobres, el grado de satisfacción declarado es significativamente inferior al de los países ricos, y dentro de los países la relación positiva entre ingreso y satisfacción es significativa, primordialmente en los niveles más bajos de ingreso relativo. En el caso de las personas que se sitúan en los tramos medio y superior de la distribución del ingreso, las variaciones de éste explican menos del $2 \%$ de la variación del nivel de satisfacción declarado (Frank, 1997, pp. 1834-1835, citando a Diener y Diener, 1995); tras concluir que, en promedio, el grado de satisfacción dentro de un país no está significativamente relacionado con el ingreso en el tiempo, Frank hace gran hincapié en el estatus relativo como fuente de respeto y factor determinante del bienestar.

Las conclusiones sobre la escasa importancia del ingreso como factor determinante del bienestar societal, que son sorprendentes al menos para algunos economistas, tal vez no sean más que una de las razones para reexaminar la base conceptual de la política en materia de pobreza. El sentido de pertenencia a una comunidad obviamente es importante para el bienestar humano, pero todavía no se ha incorporado como factor en el debate de política económica. Lo mismo puede decirse del "capital social", esto es, la capacidad de trabajar eficazmente con otros. En evaluaciones de la pobreza con participación de los afectados se ponen al descubierto algunos de los factores que influyen en el bienestar y el sentido de privación que experimentan los encuestados. En su estudio sobre la República de Guinea, Shaffer (1998) encontró que no obstante que las estadísticas de consumo no revelaban privaciones relativas de las mujeres respecto de los hombres, ponían claramente de manifiesto otros dos aspectos que afectaban desproporcionadamente a las mujeres - la carga de trabajo y la falta de autoridad para tomar decisiones o exigir respeto.

La combinación de evidencia que apunta a que al ganar más la persona efectivamente mejora su situación respecto de sus homólogos que ganan menos, pero que al aumentar los ingresos de todos a lo largo del tiempo no se produce un efecto similar en el grado medio de felicidad declarada, es compatible con la idea general de que el bienestar de las personas depende de su estatus relativo, que en las sociedades modernas se ve afectado por el ingreso y la capacidad de consumo relativos. Dadas estas actitudes, las privaciones — que son la razón por la cual la pobreza interesa como problema - pueden aliviarse principalmente, y en el caso extremo exclusivamente, disminuyendo la desigualdad 
de ingresos. Sin embargo, como lo han subrayado numerosos autores, esta situación denota algo más fundamental: que la sociedad es disfuncional, ya que mientras defina la satisfacción en términos relativos, no hay manera de lograr que mejore la situación de todos. El avance de las personas que ahora se encuentran más próximas en estatus a quienes estaban por encima de ellas es una pérdida para estas últimas, que ya no pueden disfrutar de una sensación de superioridad que les producía satisfacción. Las sociedades menos individualistas y competitivas, y con más sentido de comunidad o de sociedad, pueden beneficiarse mucho más del progreso económico. En síntesis, la evidencia empírica de los países desarrollados indica que el cambio de actitudes posiblemente sea más importante que el crecimiento económico y que sin el primero, el segundo posiblemente carezca de importancia. Si bien es cierto que esto es menos aplicable a los países en desarrollo, será también un problema en ellos si repiten los modelos de conducta de los países ricos. Uno de los objetivos clave de una sociedad debería ser reducir el componente de "suma cero" de lo que produce satisfacción a las personas.

Cabe preguntarse qué significado tiene, dadas las actitudes actuales, la evidencia parcial y hasta cierto punto desconcertante sobre los factores que determinan el bienestar humano, en cuanto a sus consecuencias prácticas para la política económica. Lo que resulta más sorprendente es el papel incierto del ingreso. En su sentido literal, los resultados parecen sugerir que, al reflexionar sobre la pobreza y el bienestar, deberíamos atribuir mucho menos importancia al crecimiento económico y que la política socioeconómica debería prestar mucho más atención que hasta ahora al empleo (en el caso de quienes lo desean), a las condiciones de empleo (incluso a evitar el exceso de trabajo), a la distribución del ingreso, a la seguridad económica, al respeto por la persona y al sentido de comunidad. Sea cual fuere el significado básico del poco peso atribuido al ingreso, hay razones para creer que la mayoría de los demás correlatos de la felicidad se relacionan y se apoyan recíprocamente. Desde luego, una de las fuentes directas principales, si no la principal, de los elevados niveles de desigualdad de ingresos es la falta de demanda de trabajadores menos calificados, lo que tiende a producir desempleo del tipo más crónico y socialmente más costoso, junto con inseguridad económica y la angustia que la acompaña. La desigualdad de ingresos también se relaciona con una distribución desigual del capital físico y humano. Estas desigualdades relacionadas entre sí son causa directa del menor respeto por quienes se encuentran en el tramo inferior de la pirámide de ingreso, riqueza, educación, calificación, etc. Naturalmente, si se logra disminuir las desigualdades se reduce el número de personas que reciben menor respeto de los demás, por mucho que éstos sigan definiendo el estatus y el respeto sobre la base del ingreso, la riqueza y la educación. En realidad, cuando estas variables no separen tanto a las personas, lo más probable es que la definición societal de las fuentes de respeto varíe en sentido favorable. Cuando hay menos diferencias entre las personas, el sentido de comunidad está más desarrollado y sus efectos son positivos.

Como se dijo, en los últimos cincuenta años no se ha observado una tendencia general positiva de la distribución del ingreso o del gasto y últimamente en demasiados países se ha dado el modelo opuesto. De ello se desprende que un indicador de la pobreza que atribuya suficiente ponderación al ingreso relativo revelará que a lo largo del tiempo, en especial en los últimos dos decenios, se ha avanzado poco o nada. Así pues, dadas las implicaciones tan diferentes de los indicadores de pobreza alternativos que se han sugerido, urge dilucidar cuáles son más significativos y quizá identificar alguna combinación de ellos que sea técnicamente defendible y que pueda atender debidamente a cada uno de los factores que determinan las privaciones y las satisfacciones que perciben las personas. La mayoría de los indicadores que utilizan los economistas son más bien ingenuos en sus supuestos implícitos sobre el origen de las percepciones individuales de privación, pero hasta ahora se ha hecho muy poco por incorporar elementos subjetivos en los indicadores compilados por los organismos internacionales y por la mayoría de los investigadores económicos.

Un problema complejo que aún queda por abordar eficazmente es el de identificar desigualdades dentro la familia y conocer la forma en que pueden haber evolucionado con el tiempo. Si el incremento de los ingresos, la urbanización y otros procesos de desarrollo en general han traído consigo avances en la igualdad de los sexos en la familia, habría que concluir que la distribución del gasto entre las personas, medida correctamente, ha evolucionado más positivamente que las estimaciones corrientes, que parten de la base de la desigualdad dentro de las familias. La forma en que ha evolucionado el bienestar subjetivo es menos clara; por ejemplo, si las expectativas de las mujeres se han elevado con más rapidez que su posición relativa, la distribución del bienestar subjetivo podría apuntar en dirección opuesta a la del gasto. 


\section{V}

\section{¿Cuáles son las mejores estrategias? ${ }^{14}$}

Así pues, los mecanismos de política más eficaces para aliviar la pobreza dependen mucho de lo que se entienda por pobreza. Es más fácil determinar cuál es la estrategia óptima cuando el concepto operativo es la pobreza absoluta, definida en función del ingreso o del gasto per cápita. En este caso, es evidente que el crecimiento es fundamental, que el crecimiento con equidad es deseable, y que las políticas cuyo principal objetivo es la redistribución probablemente no cumplan una función muy importante. Los mecanismos más prometedores son la promoción de una tasa de ahorro elevada, de preferencia con la participación amplia de la población; la inversión en capital humano, en especial en los grupos que van a la zaga en esta materia; el cambio tecnológico, con un serio esfuerzo por mantener las innovaciones dentro del ámbito de las "tecnologías apropiadas"; y medidas de política en apoyo a las unidades de producción más pequeñas. En algunos países, la política de control de población o la redistribución de los activos puede contarse entre los elementos principales, y en otros, la reforma de las instituciones y la política monetaria y fiscal. Aún queda por verse si las políticas orientadas al mercado cumplirán un papel positivo o negativo.

A medida que aumente la ponderación asignada a la pobreza relativa, también definida en función del ingreso o el gasto por persona, y a las condiciones de empleo, respeto, etc., y que disminuya aquella asignada a la pobreza absoluta, las posibilidades de éxito de las diversas políticas también cambian, quizá de manera notable. Cuando lo que más preocupa es la pobreza relativa, es posible que en la mayoría de los países las mejores opciones de política sean las de apoyar a las unidades de producción más pequeñas y tratar de aumentar el capital humano de los pobres. De ser factible, la redistribución de los activos tal vez sea la opción mejor en algunos casos, y significativa en otros. El control de población puede ser importante, en especial cuando se complementa con políticas de género vigorosas, en los países en que la desigualdad

\footnotetext{
${ }^{14}$ Entre los numerosos estudios acerca de las estrategias para reducir la pobreza, cabe mencionar el informe de Shaffer a las Naciones Unidas (Naciones Unidas, 1998), el estudio preparado por Chatterjee para el Banco Asiático de Desarrollo (Chatterjee, 1995) y el trabajo de Lipton y Ravallion (1995)
}

intergéneros es importante. En estos casos el efecto de las políticas de ahorro y de tecnología es una incógnita, ya que no hay un supuesto general de que ellas puedan mejorar la distribución, por mucho que se lleven a cabo teniendo presente ese objetivo. Es poco probable que las políticas orientadas al mercado formen parte del conjunto. ${ }^{15}$ Estas políticas pueden ser nocivas en otros sentidos: la importancia que asignan a la competencia en el mercado como camino a la riqueza para las personas, empresas, regiones, etc., podría acentuar la conciencia de la gente de su posición relativa y, en consecuencia, agudizar mucho la percepción de privación de quienes se desempeñan menos bien en el mercado.

Casi todos los que estudian la pobreza dirían que si los indicadores aplicables son el ingreso y el gasto, tanto el nivel relativo como el absoluto influyen en el bienestar de una persona; habría que concluir, por lo tanto, que la estrategia óptima se situará en algún punto entre los dos puntos que se acaban de identificar, más cerca del primero si lo que importa más es la pobreza absoluta y más cerca del segundo si pesa más la pobreza relativa.

El aspecto de la posición relativa que puede tenerse en cuenta más fácilmente al medir el cambio es el ingreso relativo. Sin embargo, como se dijo, el bienestar de una persona también depende de la forma en que es tratada por los demás y de la sensación conexa de inferioridad social, la falta de acceso a apoyo sicológico, etc. El trato discriminatorio de las mujeres, los ancianos, los niños o los grupos étnicos minoritarios también es importante para la pérdida de bienestar. Por lo tanto, la medida del bienestar societal debería tener en cuenta directamente estas formas de desigualdad. Si bien es cierto que algunas sociedades han avanzado en la solución de la desigualdad entre razas o entre género, es posible que los problemas relacionados con la vejez se tornen más severos a medida que las personas vivan hasta una edad avanzada y que disminuya la responsabilidad de la familia por ese grupo etario.

\footnotetext{
${ }^{15}$ Este es un análisis muy general. Entre las reformas compatibles con una orientación de mercado, hay algunas que probablemente empeoren la distribución del ingreso y otras que seguramente han de mejorarla.
} 
La tarea de formular políticas vuelve a alterarse y quizá se torne más compleja cuando la pobreza se define de una manera más subjetiva que tiene en cuenta las ventajas de los sentimientos de comunidad y de seguridad (que no se consideran en las cifras de ingreso absoluto o relativo, pese a que se reflejan bastante bien en el comportamiento de mercado de las personas, a través de compra de seguros, aversión al riesgo en materia de inversión, etc.). Sin una percepción clara de las preferencias societales en cuanto a lo que es una "buena vida" (reconociendo que percepciones de esta índole dependen en cierta medida de la experiencia pasada y actual) es imposible pronunciarse categóricamente acerca de lo que constituiría una política acertada. Está claro, sin embargo, que al partir de esta definición más subjetiva de pobreza la política diferirá de lo que sería si los indicadores utilizados fuesen el ingreso y el gasto, que pueden medirse objetivamente.

El deseo de seguridad no es necesariamente incompatible con la necesidad de aventura; las sociedades tienen que buscar formas adecuadas de conciliar estas metas. Si tener acceso a oportunidades es más importante para lograr satisfacción que el éxito ex post en términos económicos, esto también altera la forma en que deberían medirse los resultados económicos globales y el grado de desigualdad de una sociedad. Por desgracia, las implicaciones de política concretas de estos componentes del bienestar social son por el momento poco claras, pues no se ha evaluado detenidamente lo que podrían significar en la práctica.

(Traducido del inglés)
Akyüz, Yilmaz (1995): Financial liberalization in developing countries: Keynes, Kalecki and the Rentier, Poverty, Prosperity and the World Economy: Essays in Memory of Sidney Dell, Gerry Helleiner, Shalen Abrahamian y otros (comps.), Portland, Book News, Inc.

Banco Mundial (1993): The East Asian Miracle: Economic Growth and Public Policy, Nueva York, Oxford University Press. (1997): World Development Review 1997, Washington, D.C.

(1999): World Development Review 1998-99: Knowledge for Development, Washington, D.C.

Behrman, Jere R. y Anil B. Deolalikar (1993): Unobserved household and community heterogeneity and the labor market impact of schooling: a case study for Indonesia, Economic Development and Cultural Change, vol. 41, $\mathrm{N}^{\circ} 3$, Chicago, abril

Berry, Albert (1998a): The impact of globalization and information technology on Latin America, Globalization, Growth, and Marginalization, A.S. Bhalla (comp.), Londres, The Macmillan Press.

(1998b): Las tareas de la pequeña y mediana empresa en América Latina, Revista de la CEPAL, número extraordinario (LC/G.2037-P), Santiago de Chile, octubre.

Berry, Albert y Frances Stewart (1997): Market liberalization and income distribution: the experience of the 1980s, Global Development Half a Century After Bretton-Woods, Roy Culpeper, Albert Berry y Frances Stewart (comps.), Londres, The Macmillan Press.

Berry, Albert y John Serieux (2002): Riding the Elephants: World Economic Growth and Income Inequality at the End of the 20th Century (1980-2000), Toronto, Centro de Estudios Internacionales, Universidad de Toronto.

Berry, Albert, Francois Bourguignon y Christian Morrisson (1983): Changes in the world distribution of income between 1950 and 1977, Economic Journal, junio.

Bloom, David E. y Jeffrey G. Williamson (1998): Demographic transition and economic miracles in East Asia, World Bank Economic Review, vol. 12, № 3, septiembre.

Brightman, R.A. (1987): Conservation or resource depletion: the case of the boreal forest algonquians, The Question of the Commons, B.J. Mckay y J.M. Acheson (comps.), Tucson, The University of Arizona Press.
Chatterjee, Shiladitya (1995): Growth, Structural Change and Optimal Poverty Interventions, Occasional Papers $\mathrm{N}^{\circ} 13$, Manila, Banco Asiático de Desarrollo.

Deininger, Klaus (1999): Making negotiated land reform work: initial experience from Colombia, Brazil and South Africa, World Development, vol. 27, № 4, Amsterdam y Nueva York.

Di Tella R., R. MacCulloch y A. J. Oswald (1996): The Macroeconomics of Happiness, Oxford y Warwick, inédito.

Diener, Ed y Carol Diener (1995): The wealth of nations revisited: income and the quality of life, Social Indicators Research, vol. 36.

Dore, Ronald (1976): The Diploma Disease: Education, Qualification and Development, Berkeley, University of California Press.

Easterlin, R. (1974): Does economic growth improve the human lot? Some empirical evidence, Nations and Households in Economic Growth: Essays in Honor of Moses Abramovitz, Paul A. David y Melvin W. Reder (comps.), Nueva York y Londres, Academic Press.

Evenson, Robert (1975): Technology generation in agriculture, Agriculture in Development Theory, Lloyd G. Reynolds (comp.), New Haven, Yale University Press.

Faini, Riccardo, Fernando Clavijo y Abdel Senhadji-Semlali (1992): The fallacy of composition argument: is it relevant for LDC's manufactures exports?, European Economic Review, vol. 36.

Feder, Gershon (1983): On exports and economic growth, Journal of Development Economics, vol. 12.

Frank, Robert (1997): The frame of reference as a public good, Economic Journal, vol. 107, noviembre.

Fry, Maxwell J. (1988): Money, Interest, and Banking in Economic Development, Baltimore y Londres, The Johns Hopkins University Press.

Grosh, Margaret (1995): Five criteria for choosing among poverty projects, Coping With Austerity: Poverty and Inequality in Latin America, Nora Lustig (comp.), Washington, D.C., The Brookings Institution.

Hamada, Koichi y Jagdish Bhagwati (1975): Domestic distortions, imperfect information and the brain drain, Journal of Development Economics, vol. 2, $\mathrm{N}^{\mathrm{o}} 3$, septiembre.

Hirsch, F. (1976): The Social Limits of Growth, Cambridge, Harvard University Press.

Ho, Samuel P.S. (1980): Small-Scale Enterprise in Korea and Taiwan, World Bank Staff Working Paper, No 384, Washington D.C., Banco Mundial. 
James, J. (1998): Information technology, globalization, and marginalization, Globalization, Information Highway, and Marginalization, A.S. Bhalla (comp.), Londres, The Macmillan Press.

Kant, Shashi (1996): The Economic Welfare of Local Communities and Optimal Resource Regimes for Sustainable Forest Management, tesis de doctorado, Toronto, Universidad de Toronto.

Kant, Shashi y Albert Berry (1999): Path Dependence, Multiple Equilibria, and Adaptive Efficiency in Forest Regimes in India, Toronto, inédito.

Kuo, Shirley W.Y. (1983): The Taiwan Economy in Transition, Boulder, Colorado, Westview Press.

Kuznets, Simon (1955): Economic growth and income inequality, American Economic Review, vol. 45, $\mathrm{N}^{\circ} 1$.

Levy, Brian, Albert Berry y Jeffrey B. Nugent (1999): Fulfilling the Export Potential of Small and Medium Firms, Norwell, Massachusetts, Kluwer Academic Publishers.

Lipton, Michael (1993): Land reform as unfinished business: the evidence against stopping, World Development, vol. 21, $\mathrm{N}^{\circ} 4$, abril.

Lipton, Michael y Martin Ravallion (1995): Poverty and policy, Handbook of Development Economics, Jere Behrman y T.N. Srinivasan (comps.), vol. 3B, Amsterdam, Elsevier Science B.V.

Londoño, Juan Luis y Miguel Székely (1998): Sorpresas distributivas después de una década de reformas, Revista de Economía Política, vol. extraordinario.

Maddison, Angus (1992): The Political Economy of Poverty, Equity, and Growth: Brazil and Mexico, Oxford, Banco Mundial, Oxford University Press.

Maizels, Alfred (1994): The continuing commodity crisis of developing countries, World Development, vol. 22, № 11.

Mason, Andrew (1988): Saving, economic growth, and demographic change, Population and Development Review, vol. 14, $\mathrm{N}^{\circ} 1$.

Masson, Paul R., Tamim Bayoumi y Hossein Samiai (1998): International evidence on the determinants of private savings, World Bank Economic Review, vol. 12, No 3, septiembre.

Morduch, Jonathan (1998): Does Microfinance Really Help the Poor? New Evidence from Flagship Programs in Bangladesh, Research Program in Development Studies, Princeton, Universidad de Princeton, borrador.

Morley, Samuel (2000): Distribution and Growth in Latin America in An Era of Structural Reform, documento preparado para la Conferencia sobre los efectos de la globalización en la pobreza y en la desigualdad de los ingresos de los países en desarrollo, París, 30 de noviembre al 1 de diciembre.

Naciones Unidas (1998): Poverty Reduction Strategies: A Review, Nueva York.

Ocampo, José Antonio (2001): Agricultura y desarrollo rural en América Latina, Desarrollo rural en América Latina y el Caribe ¿la construcción de un nuevo modelo?, María Beatriz de A. David (comp.), Bogotá, D.C., CEPAL/Alfaomega.

(2002): Lights and Shadows in Latin American Structural Reforms, documento presentado en la Conferencia en honor al Profesor Albert Berry, Toronto, Universidad de Toronto, 19 y 20 de abril.

Oswald, Andrew (1997): Happiness and economic performance, Economic Journal, vol. 107. Londres.

Pritchett, Lant (1996): Where Has All the Education Gone?, Policy Research Working Paper, $N^{\circ}$ 1581, Washington, D.C., Banco Mundial.

Ranis, Gustav (1978): Equity with growth in Taiwan: how 'special' is the 'special' case?, World Development, vol. 6, $\mathrm{N}^{\circ} 3$, marzo.

Robbins, D. (1995): Trade and industrial policy reform, Handbook of Development Economics, Jere Behrman y T.N. Srinivasan (comps.), vol. B, Amsterdam.

(1996): Evidence on Trade and Wages in the Developing World, OECD Development Centre Technical Paper, $\mathrm{N}^{\circ} 119$, París, diciembre.

Rodrik, Dani (1995): Why is there Multilateral Lending?, NBER Working Papers, $\mathrm{N}^{\circ}$ 5160, Cambridge, Massachusetts, National Bureau of Economic Research.

Rosenzweig, Mark (1999): Schooling, economic growth, and aggregate data, Development, Duality, and the International Economic Regime: Essays in Honor of Gustav Ranis, Gary Saxonhouse y T.N. Srinivasan (comps.), Ann Arbor, The University of Michigan Press.

Santor, Eric (1999): Microfinance: A Review of Theory, Practice and Impact, documento preparado por el Departamento de Economía de la Universidad de Toronto, Toronto.

Schultz, Paul (1989): Education investments and returns, Handbook of Development Economics, Hollis Chenery y T.N. Srinivasan (comps.), vol. 2, Amsterdam, Países Bajos.

Schultz, Theodore W. (1961): Investment in human capital, American Economic Review, vol. 51, $\mathrm{N}^{\mathrm{o}}$ 1, marzo.

Scitovsky, Tibor (1976): The Joyless Economy, Oxford, Oxford University Press.

Serieux, John y Albert Berry (1999): Convergence, divergence or no-vergence? Recent trends in world income distribution, 1980-1995, inédito.

Shaffer, Paul (1998): Gender, poverty and deprivation: evidence from the Republic of Guinea, World Development, vol. 26, № 12 .

Stallings, Barbara y Wilson Peres (2000): Crecimiento, empleo y equidad: el impacto de las reformas económicas en América Latina y el Caribe, Santiago de Chile, Fondo de Cultura Económica/Comisión Económica para América Latina y el Caribe (CEPAL).

UNCTAD (Conferencia de las Naciones Unidas sobre Comercio y Desarrollo) (1996): World Economic and Social Survey 1996, Nueva York.

Whitehead, C. (1996): "Chronic fiscal stress and the reproduction of poverty and inequality in Latin America", in V. BulmerThomas (ed.), The New Economic Model in Latin America and its Impact of Income Distribution and Poverty (Basingstoke: Macmillan/New York: St. Martin's Press, in association with Institute of Latin American Studies, London, 1996). 
